\title{
The role of $\mathrm{B} 7$ family costimulatory molecules and indoleamine 2,3-dioxygenase in primary Sjögren's syndrome and systemic sclerosis
}

\author{
Nóra Legány ${ }^{1} \cdot$ László Berta $^{2} \cdot$ László Kovács $^{1} \cdot$ Attila Balog $^{1} \cdot$ Gergely Toldi $^{2}$
}

(C) Springer Science+Business Media New York 2016

\begin{abstract}
B7 costimulatory molecules are present on antigenpresenting cells (APCs) and influence intracellular expression of indoleamine 2,3-dioxygenase (IDO), a molecule with important immunoregulatory functions. We determined the frequency of activated (CD11b+) monocytes expressing B7-1, $\mathrm{B} 7-2, \mathrm{~B} 7-\mathrm{H} 1$, and $\mathrm{B} 7-\mathrm{H} 2$ molecules, and that of $\mathrm{CD} 3+$ and CD4+ T cells expressing the corresponding CD28, CTLA-4, PD-1, and ICOS receptors in peripheral blood samples of 20 healthy adults and $9 \mathrm{SSc}$ and $15 \mathrm{pSS}$ patients using flow cytometry. We also examined the intracellular expression of IDO. The expression of CD28 was lower in both SSc and pSS patients. The frequency of CTLA-4 was increased in pSS. The expression of ICOS, a stimulator of $\mathrm{T}$ cell activation, was elevated in pSS, but not in SSc, while that of its corresponding costimulatory molecule, B7-H2, was strongly decreased in SSc compared to controls. The frequency of PD-1 expressing $\mathrm{T}$ lymphocytes was decreased in both pSS and SSc. The frequency of IDO-expressing APCs, as well as intracellular IDO content in T cells was higher in pSS than in controls. Our investigation identified a number of differences in B7 costimulation between SSc and pSS patients which may play a role in the distinct pathogenesis and clinical features of these autoimmune disorders.
\end{abstract}

Keywords Antigen presentation $\cdot$ B $7 \cdot$ ICOS $\cdot$ Indoleamine 2,3-dioxygenase

Gergely Toldi

toldigergely@yahoo.com

1 Department of Rheumatology, University of Szeged, Szeged, Hungary

2 First Department of Obstetrics and Gynecology, Semmelweis University, Baross u. 27, Budapest H-1088, Hungary

\section{Introduction}

Antigen presentation and costimulation are the initial steps in the activation of $\mathrm{T}$ cells, and the nature and intensity of costimulatory signals are key determinants of the coordination of pro- and anti-inflammatory events.

B7 costimulatory molecules are expressed on antigenpresenting cells (APCs), including dendritic cells and monocytes, and are important regulators of $\mathrm{T}$ cell activation, as well as of cytokine production in $\mathrm{T}$ helper cells. Upon the engagement of the $\mathrm{T}$ cell receptor (TCR), the costimulatory signal from B7-1 (CD80) or B7-2 (CD86) via CD28 induces the production of IL-2 in T cells, thus protecting them from apoptosis and anergy. Both the TCR and CD28 are constitutively expressed by most naive $\mathrm{T}$ cells, enabling them to respond to the antigen being presented [1]. Without costimulation, the signal from the TCR induces the tolerance of T cells to their cognate antigen instead of being activated [2].

Nevertheless, B7 family members mediate not only stimulatory, but also inhibitory effects on T cells [1]. Engagement of the TCR induces the phosphorylation of cytotoxic T lymphocyte antigen 4 (CTLA-4), resulting in its stabilization on the cell surface. Thereby, CTLA-4 will compete with CD28 for B7 binding, and because its affinity is higher than that of CD28, it will block the costimulatory signal, and prevent further IL-2 production [3].

Another B7 family member, B7-H1 or PD-L1, has predominantly, but not exclusively, inhibitory effects on T cells. Its inhibitory function is carried out by signaling through the programmed death-1 receptor (PD-1), inducing apoptosis or anergy of self-reactive T cells [4]. Genetic deletion of PD-1 results in severe autoimmunity due to the loss of peripheral tolerance of self-reactive T cells [5].

B7-H2 or ICOSL serves as the ligand for inducible costimulator of T cells (ICOS), and promotes T cell activation, 
differentiation, and effector responses [6]. Costimulation through ICOS can augment induction of both Th1 and Th2 cytokines, including IL-4, IL-10, and INF- $\gamma$ [7].

Besides the initiation of signal transduction in $\mathrm{T}$ lymphocytes, ligation of CD80 and CD86 may back-signal into the APC and modulate their function [8]. For instance, reverse signaling through $\mathrm{CD} 80$ and $\mathrm{CD} 86$ after ligation by a soluble form of CTLA-4 was shown to upregulate the tryptophan (TRP) catabolic enzyme, indoleamine 2,3-dioxygenase (IDO) [9]. In the first steps of the kynurenine (KYN) pathway, TRP is transformed into KYN by IDO. KYN is then further metabolized by different enzymes. The local depletion of TRP and the production of proapoptotic TRP metabolites of the KYN pathway, such as 3-hydroxyanthranilic acid and quinolinic acid, are among the mechanisms potentially responsible for the immunosuppressive effects of IDO [10]. $\mathrm{KYN}$ and its metabolites suppress effector $\mathrm{T}$ cell function and facilitate their differentiation to regulatory $\mathrm{T}$ cells [11]. Since the TRP metabolic pathway is activated by proinflammatory stimuli, the anti-inflammatory effect of KYN metabolites provides a feedback mechanism in the downregulation of the immune response (Fig. 1).

Primary Sjögren's syndrome (pSS) is characterized by lymphocytic infiltration of the salivary and lacrimal glands, leading to the destruction of exocrine glands, but the autoimmune inflammatory process often involves other organs too. Systemic sclerosis (SSc), also termed scleroderma, is characterized by autoimmune activation, microvascular injury, and fibrosis in multiple organs, including the skin, lung, heart, and gastrointestinal tract. Being chronic systemic autoimmune diseases, permanent activation of the adaptive immune system is obvious in both pSS and SSc $[12,13]$. The recognition of the outstanding importance of the costimulatory regulation in many autoimmune diseases and in tumor-immunology has lead to highly effective targeted therapies in both fields [14-16].

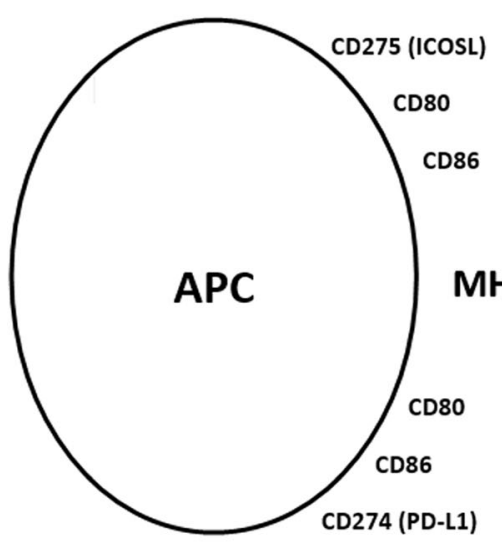

Fig. 1 B7 co-stimulatory molecules expressed on antigen presenting cells (APCs), and their stimulatory or inhibitory ligands on T cells. $M H C$ major histocompatibility complex, TCR T cell receptor, ICOS
However, data on the alterations in the expression of costimulatory molecules and their receptors in pSS and SSc are very limited. We hypothesize that the degree and the balance of the expression of the components of the B7 family member molecules and the consequent alterations in the IDO pathway may significantly determine the differences observed in the immunopathogenesis of autoimmune rheumatic disorders, included in pSS and SSc. In this study, we therefore aimed to investigate the prevalence of a major APC subset, $\mathrm{CD} 11 \mathrm{~b}+$ activated monocytes expressing CD80, CD86, PD$\mathrm{L} 1$, and ICOSL costimulatory molecules, and that of CD3+ T cells and CD4+ T helper cells expressing CD28, CTLA-4, PD-1, and ICOS, as well as the expression of IDO in these cell types in pSS and SSc.

\section{Materials and methods}

\section{Patients}

We enrolled 15 patients with pSS, 9 patients with SSc, and 20 age- and gender-matched healthy individuals. Demographic and laboratory data are summarized in Table 1.

pSS patients fulfilling American European Consensus Group (AECG) 2002 [17] or American College of Rheumatology (ACR) 2012 classification criteria [18] were included. The following clinical symptoms occurred in pSS patients' history: sicca syndrome (100\%), labial salivary gland biopsy positivity for focal lymphocytic sialadenitis with focus score $\geq 1(80 \%)$, blood cytopenia (anemia or leukopenia) $(87 \%)$, arthritis $(67 \%)$, vasculitis (53\%), anti-SSA/SSB antibody positivity (100\%), low C3/C4 complement level (47\%), renal involvement (13\%), fever and night sweats (20\%), lymphadenopathy (20\%), weight loss (33\%), myositis (7\%), and peripheral neuropathy (20\%). The EULAR Sjögren's syndrome disease activity (ESSDAI) scores were calculated at the

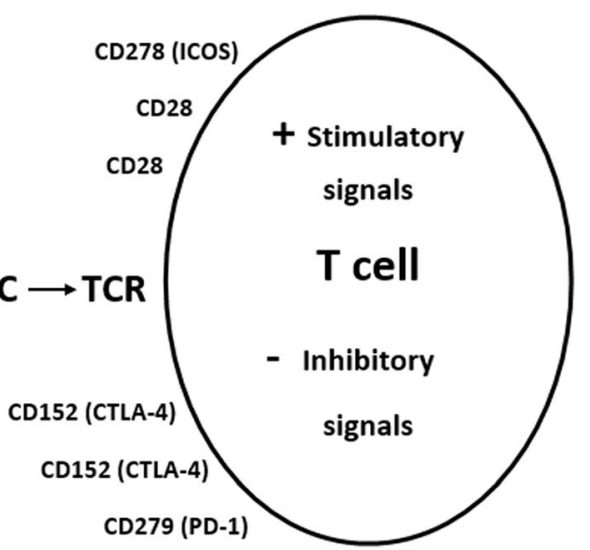

inducible costimulator, ICOSL inducible costimulator ligand, CTLA-4 cytotoxic T lymphocyte antigen-4, $P D-1$ programmed death-1 receptor, $P D-L 1$ programmed death-1 receptor ligand 
Table 1 Clinical characteristics of patients with SSc and pSS as compared with healthy controls

\begin{tabular}{lrll}
\hline Characteristics & $\begin{array}{l}\text { Healthy } \\
n=20\end{array}$ & $\begin{array}{l}\text { SSc patients } \\
n=9\end{array}$ & $\begin{array}{l}\text { pSS patients } \\
n=15\end{array}$ \\
\hline Age (years) & $53(42-61)$ & $53(39-65)$ & $52(36-77)$ \\
Gender (male/female) & $2 / 6$ & $2 / 7$ & $1 / 14$ \\
Disease duration (years) & $8(5-12)$ & $11(1-33)$ & $5(2-14)$ \\
ESR (mm/h) & $<2$ & $24^{\mathrm{a}}(1-53)$ & $27^{\mathrm{a}}(7-55)$ \\
CRP & $5.47^{\mathrm{a}}(2.0-16.0)$ & $4.8^{\mathrm{a}}(2.0-13.8)$ \\
Autoantibody positivity: & & 15 \\
Anti-SSA and/or anti-SSB & 0 & 0 \\
Anti-Scl-70 & 5 & 0 \\
Anti-centromere & 2 & 7 \\
Antirheumatic drugs & & & 10 \\
Methyl-prednisolone & 3 & 3 \\
Chloroquin & 0 & 1 \\
Methotrexate & 1 & 2 \\
Azathioprin & & & \\
\hline
\end{tabular}

Data are expressed as median (interquartile range) for continuous variables and as number for categorical variables

$S S c$ systemic sclerosis, $p S S$ primary Sjögren's syndrome, ESR erythrocyte sedimentation rate, CRP C-reactive protein

${ }^{\mathrm{a}} p<0.05$ vs. healthy individuals time of sampling [19]. The median ESSDAI score was 2 which is consistent with low disease activity. SSc patients fulfilled the 2013 ACR/EULAR classification criteria for scleroderma [20]. Patients were further classified as those with limited cutaneous SSc or diffuse cutaneous SSc according to LeRoy et al. [21]. The following clinical symptoms occurred in SSc patients' history: Raynaud syndrome (88\%), digital ulcers $(33 \%)$, interstitial lung disease (66\%), pulmonal hypertension (33\%), and pericarditis $(22 \%)$.

Healthy controls had a negative history of rheumatic symptoms. No co-morbidities were detected in patients and controls that could have influenced the investigated parameters, nor did they take any medication that could have interfered with the measurements. Written informed consent was obtained from all subjects, and our study was reviewed and approved by the ethics committee of the institution.

\section{PBMC isolation}

Peripheral blood mononuclear cells (PBMCs) were separated by a standard density gradient centrifugation (FicollPaque, Amersham Biosciences AB, Uppsala, Sweden), by means of centrifugation for $25 \mathrm{~min}, 400 \mathrm{~g}, 22{ }^{\circ} \mathrm{C}$ from freshly drawn blood collected in lithium heparin-treated tubes (BD Vacutainer, BD Biosciences, San Jose, CA, USA). Cells were kept at $-80{ }^{\circ} \mathrm{C}$ in fetal bovine serum containing $10 \%$ DMSO until analysis.

\section{Flow cytometry}

After thawing, PBMCs were incubated for $30 \mathrm{~min}$ at room temperature in the dark with the following conjugated antibodies: PerCP-conjugated CD3, PE Cy7-conjugated CD4, PE-conjugated CD28, APC-conjugated CD152 (CTLA-4), FITC-conjugated CD278 (ICOS) and APCCy7-conjugated CD279 (PD-1)mAbs, or PerCPconjugated CD3, PE Cy7-conjugated CD11b, APCconjugated CD80 and PE-conjugated CD275 (ICOSL) mAbs, or PerCP-conjugated CD3, PE Cy7-conjugated CD11b, APC-conjugated CD86, and PE-conjugated CD274 (PD-L1) mAbs in separate tubes (BioLegend, San Diego, CA, USA). After washing, cells were fixed with fixation/permeabilization solution and treated with permeabilization buffer according to the manufacturer's instructions (eBioscience, San Diego, CA, USA). They were then stained with a mouse anti-human IDO monoclonal antibody (Millipore, USA) for $30 \mathrm{~min}$ at $4{ }^{\circ} \mathrm{C}$ in the dark. After washing, cells were stained with FITC-labeled goat anti-mouse $\operatorname{IgG}$ antibody for $15 \mathrm{~min}$ at $4{ }^{\circ} \mathrm{C}$ in the dark. After washing, cells were analyzed on a BD FACSAria flow cytometer (BD Biosciences) equipped with 488- and 633-nm excitation lasers. Data were processed using the FACSDiVa software. One hundred thousand cells were recorded. The populations of lymphocytes and monocytes were gated from PBMCs according to Forward Scatter Characteristics and Side Scatter Characteristics. 


\section{Statistics}

Data are expressed as median and interquartile range. Comparisons between sample populations were made with the Kruskal-Wallis test. Correlation analyses were performed using Spearman's signed ranked tests. $p$ values less than 0.05 were considered significant. Statistics were calculated using the GraphPad Prism software (version: 5.00, GraphPad Software Inc., La Jolla, CA, USA).

\section{Results}

Cell frequency results are summarized in Table 2.

In case of $\mathrm{T}$ lymphocytes, no difference was observed in the prevalence of CD3+ CD28+ cells in SSc or pSS samples compared with healthy controls, although there was a significant increase in SSc as compared with pSS. In the T-helper subset, the prevalence of CD4 + CD28+ T-helper cells was significantly lower in both SSc and pSS than in controls. There was no difference in the prevalence of CD3 + CTLA$4+$ cells between the study groups, but within T-helper cells, the amount of CD4 + CTLA-4+ T-helper cells was higher in
pSS compared with controls. The prevalence of CD3+ ICOS+ and CD4 + ICOS+ lymphocytes was higher in pSS than in controls.

The frequency of CD3 + PD1+ and also CD4 + PD1+ lymphocytes was lower in both SSc and pSS samples than in the controls (Fig. 2). Regarding the APCs (CD11b+ monocytes), there was no difference observed in the prevalence of CD80+, CD86+, and PD-L1 + expressing monocytes between SSc, pSS and healthy control samples. However, the frequency of ICOSL+ monocytes was lower in SSc than in controls, and higher in pSS than in SSc.

No difference was observed in the prevalence of CD3+ IDO+ lymphocytes; however, IDO MFI values in CD3+ cells were higher in pSS samples compared with controls (Fig. 3).

The prevalence of IDO+ monocytes was higher only in pSS samples compared with controls, but there was no difference in the MFI of IDO in monocytes between the study groups.

In order to explore the presence of reverse signaling via CD80 and CD86, correlation analyses were performed. No correlation was revealed between IDO-expressing $\mathrm{T}$ cells and monocytes and the prevalence of CD80+ or CD86+ monocytes.

Table 2 Frequency of the investigated cell surface and intracellular markers

\begin{tabular}{|c|c|c|c|}
\hline & $\begin{array}{l}\text { Healthy control } \\
(n=20)\end{array}$ & $\begin{array}{l}\mathrm{SSc} \\
(n=9)\end{array}$ & $\begin{array}{l}\mathrm{pSS} \\
(n=15)\end{array}$ \\
\hline $\mathrm{CD} 3+\mathrm{CD} 28+$ cells/CD3+ lymphocytes & $77.18(65.78-82.50) \%$ & $84.73(77.09-88.12) \%$ & $74.87^{\mathrm{b}}(60.60-77.43) \%$ \\
\hline CD3+ CD28+ CTLA-4+ cells/CD3+ CD28+ lymphocytes & $8735(6.463-11.73) \%$ & $9650(7.920-10.09) \%$ & $11.60(7.500-12.00) \%$ \\
\hline $\mathrm{CD} 3+\mathrm{ICOS}+$ cells/CD3+ lymphocytes & $56.35(50.36-74.92) \%$ & $74.24(69.09-82.36) \%$ & $75.08^{\mathrm{a}}(73.09-77.34) \%$ \\
\hline $\mathrm{CD} 3+\mathrm{PD}-1+$ cells/CD3+ lymphocytes & $49.83(44.79-59.85) \%$ & $20.66^{\mathrm{a}}(15.00-34.42) \%$ & $17.43^{\mathrm{a}}(13.55-20.80) \%$ \\
\hline CD4+ CD28+ cells/CD4+ lymphocytes & $97.82(96.76-99.08) \%$ & $94.54^{\mathrm{a}}(87.38-96.85) \%$ & $94.54^{\mathrm{a}}(90.70-97.02) \%$ \\
\hline CD4+ CD28+ CTLA-4+ cells/CD4+ CD28+ lymphocytes & $6340(4565-8743) \%$ & $8060(5785-9975) \%$ & $11.50^{\mathrm{a}}(9.660-12.60) \%$ \\
\hline CD4+ ICOS+ cells/CD4+ lymphocytes & $59.05(53.39-73.23) \%$ & $68.12(64.87-72.95) \%$ & $74.11^{\mathrm{a}}(70.14-78.22) \%$ \\
\hline CD4+ PD-1+ cells/CD4+ lymphocytes & $54.03(47.89-62.85) \%$ & $17.45^{\mathrm{a}}(13.32-32.59) \%$ & $12.11^{\mathrm{a}}(7440-20.46) \%$ \\
\hline $\mathrm{CD} 11 \mathrm{~b}+\mathrm{CD} 80+$ cells $/ \mathrm{CD} 11 \mathrm{~b}+$ monocytes & $54.22(19.63-68.76) \%$ & $25.48(22.75-33.12) \%$ & $29.32(21.35-52.69) \%$ \\
\hline $\mathrm{CD} 11 \mathrm{~b}+\mathrm{CD} 86+$ cells/CD11b + monocytes & $27.57(19.62-29.78) \%$ & $17.35(13.21-22.52) \%$ & $27.47(16.34-36.51) \%$ \\
\hline $\mathrm{CD} 11 \mathrm{~b}+\mathrm{PD}-\mathrm{L} 1+$ cells/CD11b + monocytes & $74.09(68.22-84.90) \%$ & $84.00(51.43-86.79) \%$ & $82.40(77.16-86.21) \%$ \\
\hline $\mathrm{CD} 11 \mathrm{~b}+\mathrm{ICOSL}+$ cells $/ \mathrm{CD} 11 \mathrm{~b}+$ monocytes & $61.23(34.52-70.04) \%$ & $32.97^{\mathrm{a}}(27.93-44.23) \%$ & $59.81^{\mathrm{b}}(51.08-84.46) \%$ \\
\hline $\mathrm{CD} 3+\mathrm{IDO}+$ cells $/ \mathrm{CD} 3+$ lymphocytes & $4500(2615-7708) \%$ & $5430(3785-7205) \%$ & $5170(3730-6510) \%$ \\
\hline IDO MFI in CD3+ IDO + cells (arbitrary unit) & $\begin{array}{c}10,400 \\
(7490-11,625)\end{array}$ & $20,737(10,012-49,270)$ & $17,857^{\mathrm{a}}(13,396-22,117)$ \\
\hline $\mathrm{CD} 11 \mathrm{~b}+\mathrm{IDO}+$ cells $/ \mathrm{CD} 11 \mathrm{~b}+$ monocytes & $2585(1.970-10.84) \%$ & $6930(4.365-13.40) \%$ & $18.46^{\mathrm{a}}(12.30-21.21) \%$ \\
\hline IDO MFI in CD11b + IDO + cells (arbitrary unit) & $\begin{array}{c}22,450 \\
(19,875-24,475)\end{array}$ & $28,278(11,670-37,101)$ & $16,588(12,338-28,590)$ \\
\hline
\end{tabular}

Data are presented as median (interquartile range)

CTLA-4 cytotoxic T lymphocyte antigen-4, ICOS inducible costimulator, $P D-1$ programmed death-1 receptor, $P D-L 1$ programmed death- 1 receptor ligand, ICOSL inducible costimulator ligand, IDO indoleamine 2,3-dioxygenase, MFI mean fluorescence intensity

${ }^{a}$ Frequencies of cells isolated from SSc and pSS patients were compared with those of cells isolated from healthy individuals, $p<0.05$

${ }^{\mathrm{b}}$ Frequencies of cells isolated from pSS patients were compared with those isolated from SSc patients, $p<0.05$ 


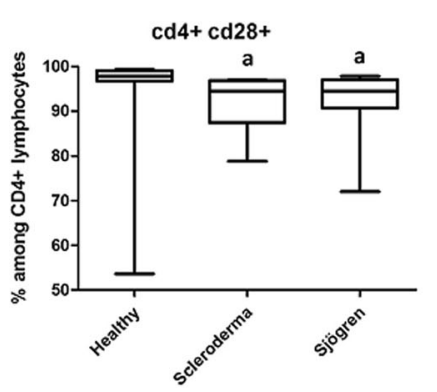

$\mathrm{cd} 3+\mathrm{cd} 28+$
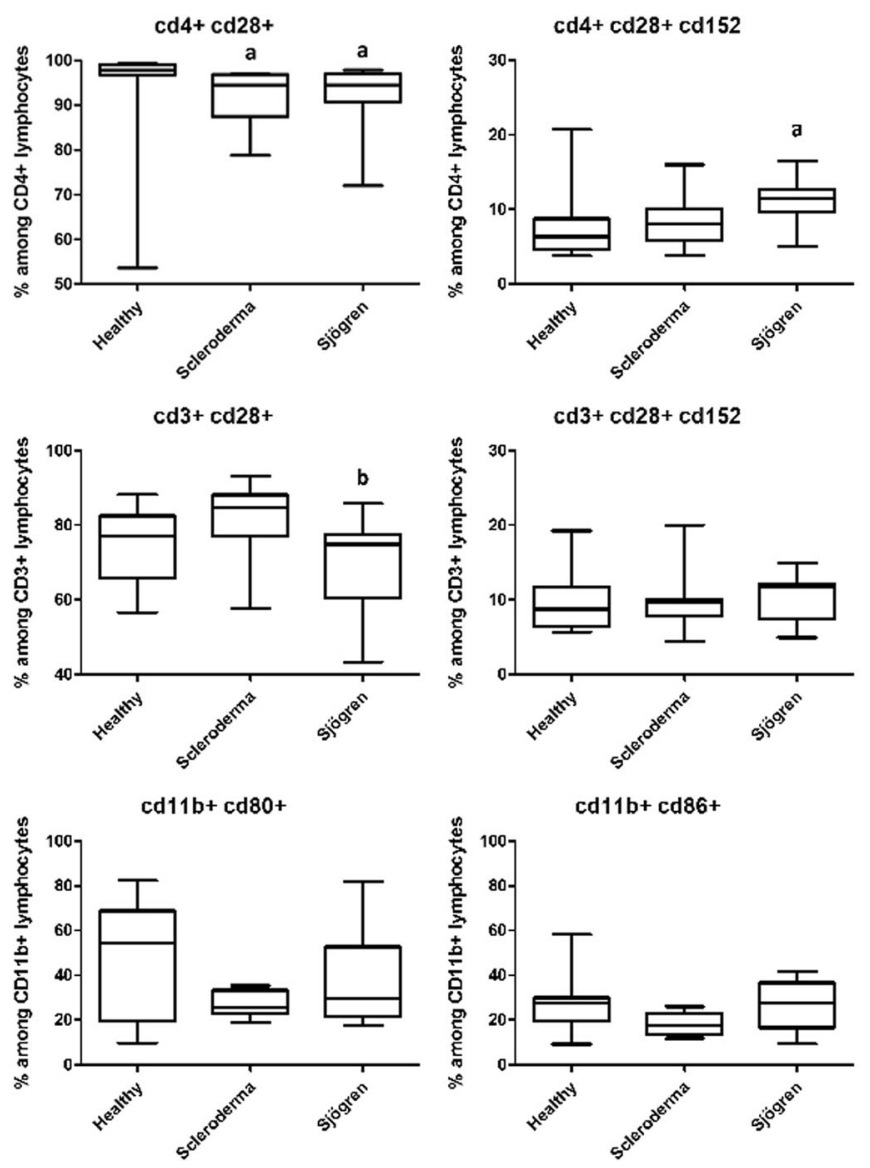

Fig. 2 Frequency of the investigated cell surface and intracellular marker-positive cells. The expression of CD28 on T helper lymphocytes and CD279 (PD-1) on T lymphocytes were lower in both SSc and pSS. The frequency of CD152 (CTLA-4) in T helper lymphocytes and CD278 (ICOS) in T lymphocytes were increased in pSS but not in SSc. The expression of CD275 (ICOSL) was strongly decreased in SSc on antigen presenting cells. SSc systemic sclerosis, $p S S$ primary Sjögren's syndrome,
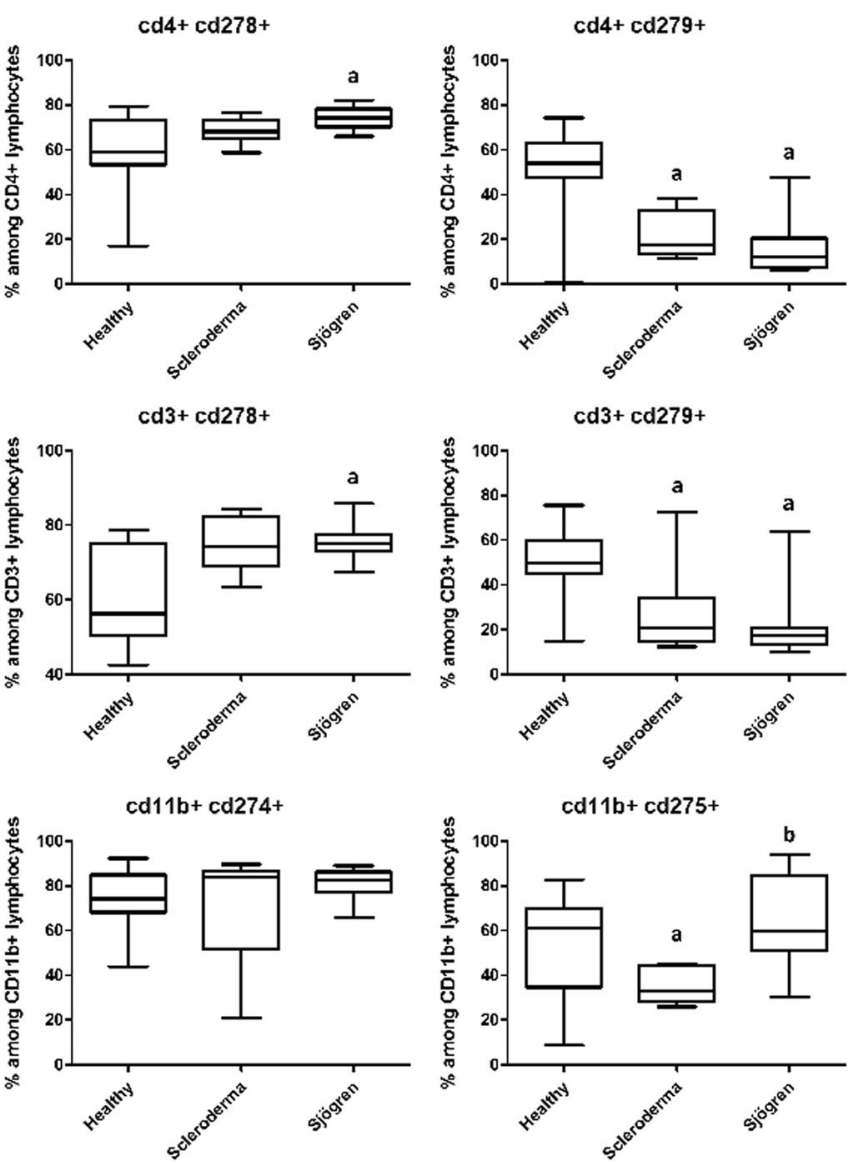

$P D-1$ programmed death-1 receptor, CTLA-4 cytotoxic T lymphocyte antigen-4, ICOS inducible costimulator, ICOSL inducible costimulator ligand. $a$ Frequencies of cells isolated from SSc or pSS patients were compared with those of cells isolated from healthy individuals, $p<0.05 . b$ Frequencies of cells isolated from $\mathrm{pSS}$ patients were compared with those isolated from SSc patients, $p<0.05$.

\section{Discussion}

The key event in the development of an autoimmune disease is the activation of effector CD4+ T cells that recognize a self peptide. There are several checkpoints that must be overcome to result in this, including the failure of central tolerance and peripheral regulation. The presentation of autoantigens will only initiate an autoimmune response if costimulatory signals overcome the checkpoints of peripheral tolerance induction. The aim of our study was to investigate the role of B7 family molecules and IDO pathway molecules on monocytes and their corresponding receptors on $\mathrm{T}$ lymphocytes in two systemic autoimmune diseases. To the best of our knowledge, our study is the first to investigate several members of costimulatory molecules at the same time in pSS and SSc.

The B7 family is one of the most important second signal mechanisms and is essential to the maintenance of the delicate balance between immune potency and suppression of autoimmunity. The importance of the $\mathrm{B} 7$ family in regulating immune responses is clear from their demonstrated role in the development of immunodeficiency, cancer, and autoimmune diseases [22]. Manipulation of the signals delivered by B7 ligands shows a great potential in the treatment of cancer or autoimmune disorders including pSS and SSc.

\section{CD80/CD86, CD28, and CTLA-4}

The upregulation of CD80/CD86 costimulatory molecules and their corresponding receptor CD28 lead to an increase in APC activity and the augmentation of $\mathrm{T}$ cell responses. In our study, the expression of CD80 and CD86 costimulatory molecules was comparable in all three study groups. At the same time, their corresponding receptor's expression of $\mathrm{CD} 28$ was lower in the T-helper subset both in pSS and SSc patients than in controls. This finding indicates a decreased costimulation via CD28 in T-helper cells in these diseases. 

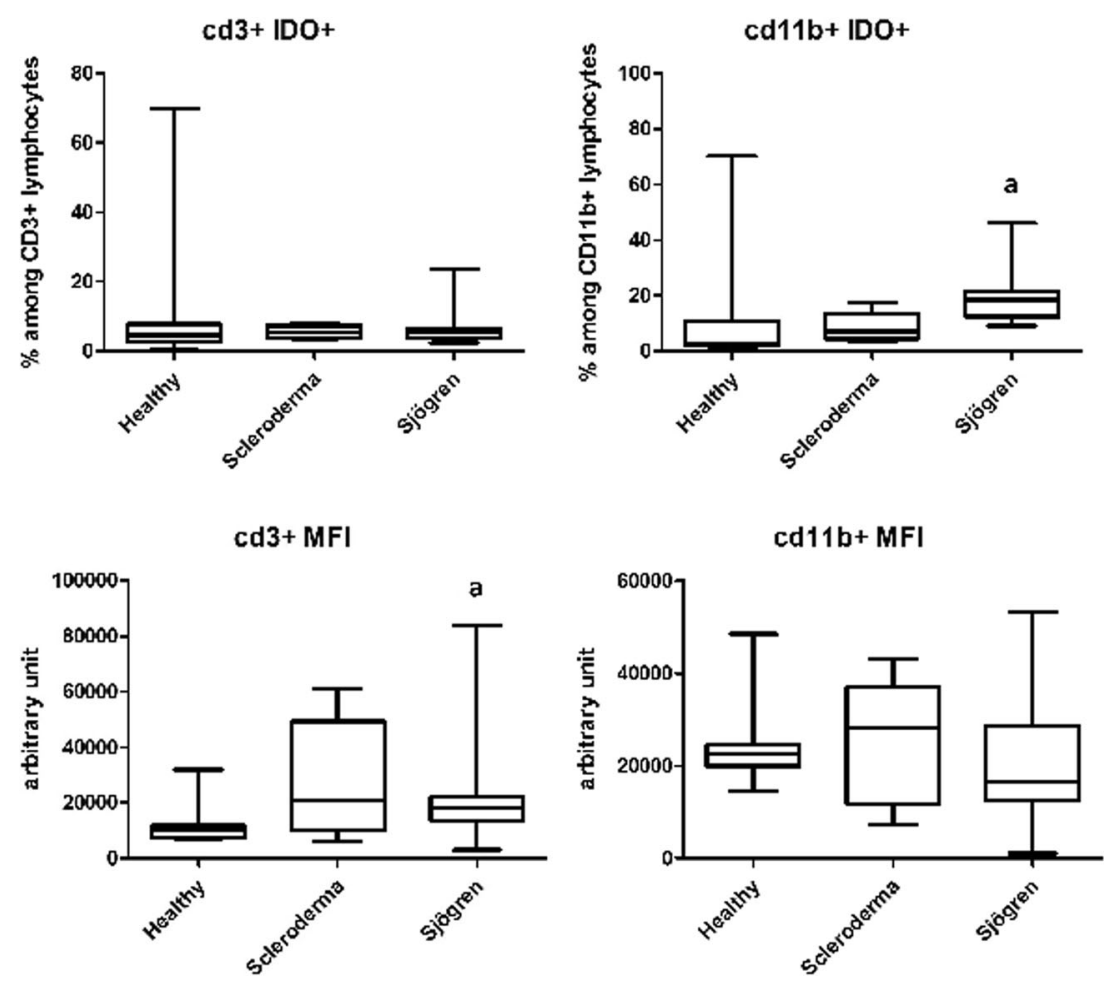

Fig. 3 IDO expression is elevated in pSS samples. While no difference was observed in IDO expression between SSc and pSS patients and controls in CD3+ lymphocytes, the frequency of IDO-expressing APCs was higher in pSS samples than in healthy controls. The intracellular IDO content (mean fluorescence intensity) was higher in CD3+ lymphocytes in pSS than in controls. There was no difference in intracellular IDO

Furthermore, in our study, the frequency of CTLA-4+ Thelper cells was higher in pSS than in controls. CTLA-4 is located on the same chromosomal region as CD28, and binds B7 more strongly. The B7 signal blockade using a CTLA-4 Ig construct leads to suppression of humoral and cell-mediated immune responses. Multiple mechanisms for CTLA-4mediated immunosuppression include competition for CD28 binding and induction of suppressive intracellular signaling pathways such as cytokine-dependent tryptophan catabolism and upregulation of IDO in B7-expressing APCs [9, 23]. Furthermore, a rapidly developing fatal lymphoproliferation and multisystemic autoimmunity was demonstrated in Ctla$4^{-/-}$mice [24].

No biological agent has yet been approved either for pSS or SSc. Abatacept is a fully human fusion molecule of IgG-Fc and CTLA-4 that modulates CD28-mediated T cell costimulation. Given the mechanism of action of abatacept and the recognized role of APCs and T and B cells in pSS and SSc, selective modulation of costimulation represents a reasonable therapeutic option. In an open-label pilot study of abatacept in active pSS, patients with recent onset of pSS and high disease activity were monitored. During the intravenous abatacept treatment, ESSDAI, ESSPRI, rheumatoid factor, and $\operatorname{IgG}$ levels decreased significantly, and fatigue and

content in APCs in SSc and pSS samples compared with healthy controls. IDO indoleamine 2,3-dioxygenase, $S S c$ systemic sclerosis, $p S S$ primary Sjögren's syndrome, APCs antigen-presenting cells, $M F I$ mean fluorescence intensity. $a$ Cells from pSS patients were compared with healthy controls, $p<0.05$.

health-related quality of life improved significantly [16]. Abatacept also induces clinical improvement in patients with severe and treatment-resistant diffuse SSc [15]. In our study, the lower level of CD28 receptors on T-helper cells in pSS and SSc and the higher level of CTLA-4+ T-helper cells in pSS suggest a "protective" status of costimulation between APCs and $T$ cells that reduces the $T$ cell-dependent autoimmune response. The enrolled patients with pSS and SSc in our study were selected randomly from our outpatient clinic. All investigated patients with $\mathrm{pSS}$ or with SSc had a low disease activity according to ESSDAI, ESSPRI, mRSS, and EScSG activity indices, respectively. All patients had relatively long disease duration, and took immunosuppressive therapy. It may be hypothesized that either the long disease duration (chronic autoimmune process) or the long-term effect of immunosuppressive agents might have lead to the desensitization or lower expression of CD28 receptors and higher level of CTLA-4 on T-helper cells in pSS and SSc.

\section{ICOS}

We have demonstrated that in $\mathrm{T}$ lymphocytes, the expression of ICOS, a stimulator of T cell activation, is elevated in pSS, but not in SSc, while that of its corresponding costimulatory 
molecule, ICOSL, was strongly decreased in SSc compared to controls. Therefore, this pathway appears to play a more dominant role in $\mathrm{pSS}$.

Accumulating evidence suggests that ICOS is instrumental in T cell-driven multi-organ inflammation in autoimmune diseases [25]. ICOSL was found to be expressed not only in APCs, but also in salivary gland epithelial cells (SGECs) in pSS. These SGECs play an important role in the differentiation of naive CD4+ T cells into follicular helper T cells by the secretion of IL-6. Interactions between ICOSL on SGECs and ICOS on follicular helper $\mathrm{T}$ cells results in enhanced IL-21 secretion by follicular helper T lymphocytes [26]. In another study, ICOSL expression by CD11c+ myeloid cells was associated with enhanced $\mathrm{T}$ cell survival in the kidneys of lupus-prone mice. Local activation of ICOS by CD11c+ myeloid cells drives organ inflammation in lupus. These results suggest an autoantibody-independent role for ICOS in selfreactive $\mathrm{T}$ cell survival in target organs [27]. According to these findings, our results point to a potential role of ICOS in the pathomechanism of pSS, potentially by means of the maintenance of a sustained stimulation of $\mathrm{T}$ cells within the target organs, including the salivary gland.

Similarly to our findings, a greater frequency of circulating $\mathrm{T}$ follicular helper ( $\mathrm{Tfh}$ ) cells express ICOS in systemic lupus erythematosus (SLE) compared to healthy controls. Furthermore, in vitro ICOS costimulation of peripheral T cells from patients with active SLE resulted in greatly enhanced IFN- $\gamma$ production relative to normal controls, and ICOS ligation preferentially induced production of isotype-switched $\alpha$ double-stranded DNA ( $\alpha$-dsDNA) antibodies during coculture with autologous B cells. In animal models, splenic ICOS+ CD4+ T cell production of IL-21 was linked to renal disease and early mortality. In a different study, DC ICOSL expression was correlated with kidney nephritis and proteinuria. As a whole, by way of enhancing autoantibody formation and accelerating organ inflammation, these data strongly suggest that ICOS plays a direct role in promoting SLE disease progression [28]. Therapeutic interventions designed to disrupt ICOS/ICOSL signaling may therefore be similarly promising in $\mathrm{pSS}$ as in SLE, taking our current findings into consideration.

\section{PD-1}

The frequency of PD-1 expressing T lymphocytes was found to be decreased both in pSS and SSc compared with controls, while there was no difference in the PD-L1 expression of APCs between the investigated study groups.

PD-1 regulates self-tolerance against many organs and prevents autoimmunity by setting at least two checkpoints that control the induction and maintenance phases of the anergic state. In addition, the innate immune system may inhibit autoreactive lymphocyte proliferation by PD-1-induced pathways [29]. Decreased frequencies of PD-1 expressing T cells may reflect deficiencies in this regulatory pathway in these two connective tissue diseases.

\section{IDO}

There is abundant evidence of immune cell hyper-activation in pSS and SSc. This raises the question of whether there is corresponding activation of the IDO pathway, in an attempt to regulate uncontrolled $\mathrm{T}$ cell responses. IDO is the first and rate-limiting step in the KYN pathway, and is thought to play a key role in immune homeostasis through depletion of tryptophan and accumulation of KYN metabolites. Inhibition of IDO activity, or knockout of the gene encoding IDO, was shown to cause an increase in the severity of collageninduced arthritis, an animal model of rheumatoid arthritis [30]. On the other hand, a higher percentage of IDOexpressing APCs was observed supported in pSS than in healthy controls [31]. Increased KYN concentration and KYN/TRP ratio, which reflects the activity of IDO, were also measured in the peripheral blood of pSS patients [32]. In our study, we found that the frequency of IDO-expressing APCs, as well as intracellular IDO content in T cells, was higher in pSS than in controls. Therefore, it is possible that the increased IDO activity tries to control the chronic autoantigen stimulation in pSS and plays a role in the counter-regulatory response.

Our results also supported that the induction of IDO activity resulting in the activation of the KYN pathway is an attractive therapeutic approach. However, further investigation of the downstream members of the KYN pathway is needed to confirm or refute this hypothesis.

A limitation of our study is the lack of data on changes in cytokine and chemokine expression related to alterations observed in the costimulatory pathways. These linked mechanisms also play a key role in the pathomechanism of pSS and SSc and need further investigation based on the current results. Another limitation may be the heterogeneity of our patient populations in terms of treatment with different immunosuppressive agents. In future studies, patients with more active disease state should also be included.

\section{Conclusion}

Our understanding of the B7 family has expanded enormously since the description of costimulatory pathways. Contemporary models of immune regulatory networks reflect this complexity. Defining the signals by which specific types of APCs and costimulatory molecules drive T cell autoimmunity, and elucidating whether these signals play a disease 
specific role, will advance our knowledge towards the development of new therapeutics that rely on disrupting T cell-APC interactions. The promise of modification of autoimmune mechanisms through pharmacologic manipulation of this pathway shows substantial potential. Furthermore, our results also suggest that the expression of costimulatory molecules differs between distinct autoimmune disorders, although a limitation of the current study is the low number of patients. Therefore, sub-classification of the investigated patients' group according to clinical characteristics (disease activity, disease duration, medication, etc.) would provide further details about the role of costimulatory pathways.

Acknowledgments Attila Balog and Gergely Toldi were supported by the János Bolyai Scholarship of the Hungarian Academy of Sciences.

\section{References}

1. Harris NL, Ronchese F. The role of B7 costimulation in T-cell immunity. Immunol Cell Biol. 1999;77:304-11.

2. Gimmi CD, Freeman GJ, Gribben JG, et al. Human T-cell clonal anergy is induced by antigen presentation in the absence of B7 costimulation. Proc Natl Acad Sci U S A. 1993;90:6586-90.

3. Chambers CA, Kuhns MS, Egen JG, et al. CTLA-4-mediated inhibition in regulation of $\mathrm{T}$ cell responses: mechanisms and manipulation in tumor immunotherapy. Annu Rev Immunol. 2001;19:56594.

4. Wang S, Bajorath J, Flies DB, et al. Molecular modeling and functional mapping of B7-H1 and B7-DC uncouple costimulatory function from PD-1 interaction. J Exp Med. 2003;197:1083-91.

5. Nishimura H, Nose M, Hiai H, et al. Development of lupus-like autoimmune diseases by disruption of the PD-1 gene encoding an ITIM motif-carrying immunoreceptor. Immunity. 1999;11:141-51.

6. Coyle AJ, Lehar S, Lloyd C, et al. The CD28-related molecule ICOS is required for effective T cell-dependent immune responses. Immunity. 2000;13:95-105.

7. Wang S, Chen L. T lymphocyte co-signaling pathways of the B7CD28 family. Cell Mol Immunol. 2004;1:37-42.

8. Petroff MG, Perchellet A. B7 family molecules regulators of the maternal immune system in pregnancy. Am J Reprod Immunol. 2010;63:506-19.

9. Grohmann U, Orabona C, Fallarino F, et al. CTLA-4-Ig regulates tryphtophan catabolism in vivo. Nat Immunol. 2002;3:1097-101.

10. Mándi Y, Vécsei L. The kynurenine system and immunoregulation. J Neural Transm (Vienna). 2012;119:197-209.

11. Filippini P, Del Papa N, Sambataro D, et al. Emerging conception inhibitors of indoleamine 2,3-dioxygenase in rheumatic disease. Curr Med Chem. 2012;19:5381-593.

12. Hansen A, Lipsky PE, Dörner T. New concepts in the pathogenesis of Sjögren syndrome: many questions, fewer answers. Curr Opin Rheumol. 2003;15:563-70.

13. Varga J, Abraham D. Systemic sclerosis: a prototypic multisystem fibrotic disorder. J Clin Invest. 2007;117:557-67.
14. Chinai JM, Janakiram M, Chen F, et al. New immunotherapies targeting the PD-1 pathway. Trends Pharmacol Sci. 2015;36:58795.

15. de Paoli FV, Nielsen BD, Rasmussen F, et al. Abatacept induces clinical improvement in patients with severe systemic sclerosis. Scand J Rheumatol. 2014;43:342-5.

16. Meiners PM, Vissink A, Kroese FG, et al. Abatacept treatment reduces disease activity in early primary Sjögren's syndrome. Ann Rheum Dis. 2014;73:1393-6.

17. Vitali C, Bombardieri S, Jonsson R, et al. Classification criteria for Sjögren's syndrome: a revised version of the European criteria proposed by the American-European Consensus Group. Ann Rheum Dis. 2002;61:554-8.

18. Shiboski SC, Shiboski CH, Criswell L, et al. American College of Rheumatology classification criteria for Sjögren's syndrome: a data-driven expert consensus approach in the Sjögren's International Collaborative Clinical Alliance cohort. Arthritis Care Res. 2012;64:475-87.

19. Seror R, Bowman SJ, Brito-Zeron P, et al. EULAR Sjögren's syndrome disease activity index (ESSDAI): a user guide. RMD Open. 2015;1:e000022.

20. van den Hoogen F, Khanna D, Fransen J, et al. 2013 classification criteria for systemic sclerosis: an American College of Rheumatology/European league against rheumatism collaborative initiative. Arthritis Rheum. 2013;65:2737-47.

21. LeRoy EC, Black C, Fleischmajer R, et al. Scleroderma (systemic sclerosis): classification, subsets and pathogenesis. J Rheumatol. 1988;15:202-5.

22. Greaves P, Gribben JG. The role of B7 family molecules in hematologic malignancy. Blood. 2013;121:734-44.

23. Rudd CE, Schneider H. Unifying concepts inCD28, ICOS and CTLA4 co-receptor signalling. Nat Rev Immunol. 2003;3:544-56.

24. Waterhouse P, Penninger JM, Timms E, et al. Lymphoproliferative disorders with early lethality in mice deficient in CTLA-4. Science. 1995;270:985-8.

25. Odegard JM, Di Placido LD, Greenwald L, et al. ICOS controls effector function but not trafficking receptor expression of kidneyinfiltrating effector T cells in murine lupus. J Immunol. 2009;182: 4076-84.

26. Gong YZ, Nititham J, Taylor K, et al. Differentiation of follicular helper T cells by salivary gland epithelial cells in primary Sjögren's syndrome. J Autoimmunity. 2014;51:57-66.

27. Teichmann LL, Cullen JL, Kashgarian M, et al. Local triggering of the ICOS coreceptor by CD11c + myeloid cells drives organ inflammation in lupus. Immunity. 2015;42:552-65.

28. Wikenheiser DJ, Stumhofer JS. ICOS Co-stimulation: friend or foe? Front Immunol. 2016;7:304.

29. Chikuma S. Basics of PD-1 in self-tolerance, infection, and cancer immunity. Int J Clin Oncol. 2016;21:448-55.

30. Williams RO. Exploitation of the IDO pathway in the therapy of rheumatoid arthritis. Int J Tryptophan Res. 2013;21:67-73.

31. Furuzawa-Carballeda J, Hernández-Molina G, Lima G, et al. Peripheral regulatory cells immunophenotyping in primary Sjögren's syndrome: a cross-sectional study. Arthritis Res Ther. 2013;15:R68.

32. Petrovaara M. RaitalaA, Uusitalo $\mathrm{H}$, et al. Mechanisms dependent on tryptophan catabolism regulate immune responses in primary Sjögren's syndrome. Clin Exp Immunol. 2005;142:155-61. 